\title{
Clinical features of the Anterior Chamber Depth
}

\author{
Miguel Angel Hernández-Delgado, MD (iD, Sara Gonzalez Godínez, MD (iD and Jorge Valdez-García, \\ MD*i \\ Tecnologico de Monterrey, School of Medicine \& Health Sciences, Institute of Ophthalmology \& Visual \\ Sciences, Monterrey, Mexico

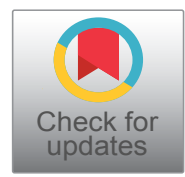

*Corresponding author: Jorge Valdez-García, MD, Institute of Ophthalmology \& Visual Sciences at Zambrano Hellion Medical Center, Av. Batallon de San Patricio No. 112 San Pedro, Nuevo Leon, Mexico, Postal code: 62670. Phone number: (+52) 81-82-59-07-79

\begin{abstract}
Purpose: To determine whether anterior chamber depth (ACD) has any clinical importance as well as the significance in various physiologic and pathologic processes.

Methods: Recollection of the most relevant data from the relationships of ACD in eye care were extracted from PubMed, SCOPUS, Google Scholar and ARVO. Forty-two total articles were used in the construct of this review.

Results: Anterior chamber depth has a direct proportional relation with axial length, corneal power and anterior chamber angle. It has an inverse proportional relation with age. Shallow anterior chamber depth was associated with and increased risk of endothelial cell density loss after phacoemulsification surgery, less ascorbic acid concentrations that translates to endothelial damage independently of surgery, angle closure glaucoma and angle closure secondary to retinal vein occlusions, trabeculectomy and tube placement complications and IOL formulae selection and refractive outcome inaccuracies. Population studies found this parameter to be different depending on the observed race and high rates of heritability, men were found to have deeper anterior chambers than women across all ethnic groups.

Conclusion: ACD plays an influential role in many physiologic and pathologic conditions. These include, IOL lens formula usage, expected refractive outcomes after phacoemulsification surgery, corneal endothelial cell loss, closed angle glaucoma, trabeculectomy and anterior chamber tube endothelial cell loss, closed angle glaucoma, trabeculectomy and anterior chamber tube processes.
\end{abstract}

\section{Keywords}

Anterior chamber geometry, Axial length, IOL formula

\section{ACD Characteristics and Modifying Factors}

Anterior Chamber Depth (ACD) plays an important role in several eye conditions, including inflammatory eye processes, keratoconus, endothelial cell density loss (ECDL), oxidative stress in endothelial cells, glaucoma surgical interventions, phacoemulsification surgery, anterior chamber dimensions, intraocular lens (IOL) formula calculations and surgical post refractive errors.

ACD corresponds to the distance between the corneal endothelium to the anterior lens capsule. Some authors may include the corneal thickness (CT) in this term, increasing approximately $0.5 \mathrm{~mm}$, corresponding to the average $\mathrm{CT}$ in $\mathrm{mm}$. Although this is not a consensus, it is usually specified as ACD-CT [1].

$A C D$ varies with age and gender. It has an inverse proportional relation with age, and directly proportional relation with the anterior chamber angle (ACA), and axial length $(A L)[2,3]$. Moreover, for every millimeter increase in $A L$ the $A C D$ distance increases by $0.07 \mathrm{~mm}$, and by every year increment in a patient's life the $A C D$ decreases by $0.005 \mathrm{~mm}$. This led to the following formulas; estimated ACD [3.339 - 0.005(age in years)] and ACD [0.07(axial length in $\mathrm{mm}$ )+1.4892] [4]. Other reported rates of ACD shallowing per year have been described, by $X u$ in the Beijing Eye Study at $9 \mu \mathrm{m} /$ year [5] Fernandez-Vigo by $10.4 \mu \mathrm{m} /$ year [6], Rufer $11.5 \mu \mathrm{m} /$ year [7], Sun by $11.9 \mu \mathrm{m} /$ year [8] Sng by $15 \mu \mathrm{m} /$ year [9] and Yan by $17 \mu \mathrm{m} /$ year [10]. The relevancy of this issue, albeit the immense variations just described, lies in the young individual that receives an IOL. Given their theoretically

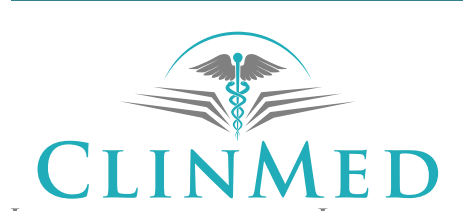

INTERNATIONAL LIBRARY
Citation: Hernández-Delgado MA, Godínez SG, García JV (2021) Clinical features of the Anterior Chamber Depth. Int J Ophthalmol Clin Res 8:125. doi.org/10.23937/2378-346X/1410125

Accepted: April 16, 2021: Published: April 19, 2021

Copyright: @ 2021 Hernández-Delgado MA, et al. This is an open-access article distributed under the terms of the Creative Commons Attribution License, which permits unrestricted use, distribution, and reproduction in any medium, provided the original author and source are credited. 
long-life expectancy, ACD decrements affect the tolerability of the lens [6].

In addition to these observations, wide ACA is associated 80 with a deeper anterior chamber (AC), longer $A L$ and higher corneal power, as proven in the Gutenberg Health Study [11]. In a cohort study comparing the AC in children (mean age $7.1 \pm 3.3$ years) and adults (73.7 \pm 7.8 years), children had significantly deeper $A C D$, longer inter scleral-spur distance and larger corneal curvatures [12].

Due to modern technology, there are several approaches in anterior chamber measurement. Naturally, some techniques are far more accurate than others. Hoffer and Savini studied which were the most accurate methods. By comparing the older of them, manual optical pachymeter, versus mode A ultrasound. And pachymetry versus partial coherence inferenciometry ( $\mathrm{PCl}$, such as IOL Master). They determined that mode $\mathrm{A}$ ultrasound gave shorter $A C D$ readings, related to the ultrasound velocity, across the different density between the cornea and aqueous humor. Optical pachymetry proved to be just as unequivocal as $\mathrm{PCl}$, existing only a minor and not statistically significant difference among them. Thus, concluding that for accurate ACD measurement, optical means such as $\mathrm{PCl}$ and standard pachymetry shall be the prefered methods in acquiring this measurement [13].

When using $\mathrm{PCl}$ devices (IOL Master 500 or IOL Master 700) versus Scheimpflug camera methods (Pentacam $A X L)$, the doubt remained if these had good agreement. In fact, the precision for these methods in acquiring ACD was just as exact. Although they may vary in the collection of other parameters such as AL (where IOL Master devices excell) or corneal curvatures (where Pentacam AXL was proven more accurate) [14].

Many other ophthalmological exploration estimations may be obtained from the anterior chamber, such as the Van Herick method for iridocorneal angle estimation. Recently, a newer 'Van Herick Plus' method was described by Sihota for peripherally measured ACD and angle [15]. Since the inferior part of the anterior chamber is more oftenly closed than the temporal or nasal section [16], a short vertical light beam straddling the inferior limbus and not reaching the pupil (to prevent miosis that may open the angle) was far more sensitive than standard Van Herick technique and it had a good correlation with anterior segment optical coherence tomography (AS-OCT): This method could be used for more accurate screening 110 methods for eyes requiring full gonioscopy for iridocorneal angle evaluation [15].

Physiological conditions may change the ACD measurement. Mete et al. found that the valsalva maneuvers reduce it by anterior displacement of iridolenticular diaphragm during this exercise [17]. Pregnancy was found to vary ACD in the third trimester versus postpartum period; these changes were not statistically significant, but the changes were attributed to $\mathrm{CT}$ change as a response to stromal water retention by hormonal effect [18]. For many years, the question remained if low birth weight was related to adulthood modifications in anterior chamber geometry. It was not until the Gutenberg Health Study provided with the database power to analyse whether this factor negatively impacted the AL and $A C D$. Contrary to the expectation, low birth weight was no associated with short ACD after adjustment with age and sex [19].

Interestingly, certain supra-physiologic activities such as space flight, leads to variations in ocular structures that are clinically important. Spaceflight-associated Neuro-ocular Syndrome (SANS) was described by the National Aeronautics Space Administration (NASA) after many observations in astronauts that spent more than 1 month in spaceflight and had developed hyperopic shifts, optic disc oedema, choroidal folds and globe flattening. After 1 year of follow up, the astronauts in this cohort presented many ocular changes. Amongst them was a significant change in $A L$ and $A C D$ that explained the hyperopic shift, optic nerve oedema and choroidal thickness was present as well. More research on zero gravity conditions and their effect on ocular physiology are needed to answer why the hyperopic shift remained for more than 1 year after the spaceflight return and if the other ocular signs and symptoms are preventable [20].

Less obvious physiologic changes and their relationships with $A C D$ are related to ascorbic acid (AA) concentrations. These vary greatly among sex and ACD. Despite AA being twenty times higher in concentration in aqueous humor than that of plasma, differences in this vitamin either defends or exposes the endothelial cells to detrimental reactive oxygen species (ROS). Higher AA concentration in the $A C$, reduces the possibility of ROS interacting and damaging many other structures such as the trabecular 140 meshwork, lens, and the rest of the corneal layers. Since the phacoemulsification surgery readily replenishes the anterior chamber volume, along with this relevant molecule, there is an incremental risk of ECDL. Albeit, it wasn't fully understood if ACD or AL played any independent role in AA concentration. Just recently, we can observe that female gender, and shallow ACD are associated with lower AA concentrations. Hence, venturing patients with these characteristics to phaco surgery may have an even greater effect in ECDL [21]. It has been theorized that a few sets of genes; SVCT1, SVCT2, and GLUT1, and their polymorphisms [22] may be responsible for this difference in aqueous humor AA concentrations in women. As well as with ACD dimensions [21,22].

\section{ACD Variations}

There are several studies that determine the normal 
parameters of ACD. Ethnic variations are an important consideration when discussing ACD. It has been extensively proven that age, sex, spherical equivalent and ethnicity have a significant effect on this parameter [23]. The heritability of ACD distance was conclusively proven by Klein et al. who conducted familial correlation and heritability in 750 subjects using the data from the Beaver Dam Eye Study cohort. Alongside ACD that was highly heritable (estimates ranging from 51 - 94\%), so did other variables such as SE, corneal curvature and AL [24].

The Beijing Eye study evaluated a total of 2985 individuals using AS-OCT. The mean ACD was $2.42 \pm 0.34$ $\mathrm{mm}$, and shallower in women than in men. The study found that shallow ACD was significantly ( $p<0.001)$ related to age, hyperopic refractive error, female versus male gender, shorter body height and optic disc area [5].

The Singapore Chinese Eye Study evaluated 1060 subjects using AS-OCT and found a mean ACD of 3.24 $\pm 0.35 \mathrm{~mm}$, greater in men (3.29 $\pm 0.36 \mathrm{~mm})$ than in women (3.19 $\pm 0.33 \mathrm{~mm})$. Women had a greater lens vault (LV), iris curvature and IOP than men. Most importantly, shallow ACD was directly influenced by age, large $\mathrm{LV}$, angle opening distance at $750 \mu \mathrm{m}$ from the scleral spur (AOD750), large $A C W$, short $A L$, grand iris area and posterior corneal arc length (PCAL). In this study, the cardinal factors determining the ACD measurement are PCAL and LV. Other variables like height (in direct disagreement to 170 the results found in the Beijing Eye Study), diabetes mellitus, iris curvature, IOP, cupto-disc ratios were not significantly associated with $A C D$ [9]. Despite this fact, diabetes is still an important risk factor to consider for its lens thickness (LT) enlargement mechanisms [25].

Chinese population has a shallower AC, smaller anterior chamber width (ACW), anterior chamber angle/ volume (ACA, ACV), independently of age and $A L$, compared with Caucasians. Thus, it is wise to consider the $A C$ characteristics in regard to ethnicity [23]. In fact, when comparing AC parameters between Chinese and Vietnamese populations, the former had an even greater $A C D$, rendering the latter with important clinical consequences, mainly related to primary angle closure [26].

Lozano et al. found in a retrospective study involving 124 subjects that the mean ACD in a Hispanic population in the northern mexican region was $2.92 \pm 0.44 \mathrm{~mm}$ and that ACD had a positive correlation with pupil diameter in a statistically significant manner [27].

\section{Surgical Considerations Regarding ACD}

Surgical interventions such as phacoemulsification techniques have an important modification effect in $A C D$. The relative change in ACD was more pronounced and statistically significant, in shorter $A L$ eyes than in normal AL or high AL [28].
ACD proved to be a key predictor for refractive outcomes in age related cataract surgery (ARCS). This measurement increases in the first two weeks after phacoemulsification cataract surgery, in a statistically significant matter. This expansion halted after the two week period. Depending on whether this change in ACD is relatively small, and hyperopic overcorrection is seen postoperatively, the contrary leading to a more myopic result. Accordingly, Ning et al. proved that there is a positive relationship between preoperative $A C D$ and postoperative refractive error. Additionally, to a significant correlation between the incidence of refractive errors depending on whether the $A L$ is $<22 \mathrm{~mm}$ or $>26$ $\mathrm{mm}$. The author also proposed two regression formulas for correctly estimating this ACD change. Although further validation is still required. These formulas are, for postoperative 200 ACD $(3.524+0.294 \times$ preoperative $A C D)$ and postoperative ACD $(3.361+0.228 \times$ [preoperative ACD + 1/2 LT]) [29].

Furthermore, shallow $A C D$ along with short $A L$ is an important risk factor for ECDL. Khalid et al. demonstrated that AL between 22-23.5 mm and ACD in the 2-3 mm range had greater mean change in corneal endothelial density after phacoemulsification surgery with IOL implantation [30]. Along with these findings, anterior chamber volume (ACV) and lens density (LD) may also be crucial factors regarding ECDL [31].

Likewise, in Descemet Stripping Automated Endothelial Keratoplasty (DSAEK) and in Descemet Membrane Endothelial Keratoplasty (DMEK), a shallow ACD creates a greater loss of endothelial cells and a deleterious effect on their long-term survival [32].

It is known that the pseudoexfoliation syndrome (PEX) gives rise to many surgical challenges, including floppy iris syndrome, glaucoma, zonular dialysis, phacodonesis, lens subluxation and less intracameral response to mydriatic agents. It was recently proposed by Gungar et al. another clinical correlation involving PEX and its effect on ACD. They compared normal AL eyes versus normal AL PEX eyes after phacoemulsification surgery, concluding there was a substantial change in ACD compared to non PEX individuals. This is paramount to consider in IOL formula selection and postoperative refractive results $[33,34]$.

Primary open angle glaucoma (POAG) and Primary angle closure glaucoma (PACG) retain an unequivocal relationship with $A C D$. Even if this either helps or promotes further deterioration. POAG patients have a greater LT and shallower ACD compared to healthy controls, independently of IOP. IOP reduction may also be achieved in lens removal, depicting its great importance in PAOG. ACD being significantly reduced in the female population and with older patients, this represents important risk factors that shall be considered. In PACG, the most well-known risk factors are Asian population, hyperopic eyes, and shallow ACD [35]. 
In cases where anterior chamber tube placement is imperative, ACD symmetry shall be carefully considered. Since the narrowest part of the AC lies in the superior portion in pseudophakic patients, and the tube-endothelial distance is directly influenced by the ACD, this parameter is essential in order to avoid further endothelial cell loss or injury. Despite this fact, most drainage tubes are placed superotemporally or in a supero-nasal position. Thus, a broader ACD is a protective factor in case of anterior chamber IOL or tube placement [36]. Following trabeculectomy, ACD decreases importantly in the first 4 postoperatives days and reaches $91 \%$ of its preoperative value at 14 days. After this 2 -week period and so forth, ACD does not in fact significantly change this procedure [37].

The anterior chamber may reduce its length with central retinal vein occlusions (CRVO), mainly by the vascular congestion of the ciliary body and an increase in posterior pole volume, this may be so extreme that the resulting displacement often leads to angle closure in shallow ACD (specially if less than $2 \mathrm{~mm}$ ) eyes or in the context or reduced AL. This exemplifies the emphasis that we shall all have in performing gonioscopy following CRVO, both for the angle closure that this may cause, and the manifestation of a neovascular glaucoma [38].

Anterior chamber modifications in keratoconus are numerous. Despite the obvious corneal changes that modify its symmetry, ACD cannot only be attributed to corneal curvature, but also limbal alterations are responsible for this parameter increment. Not only does ACD is significantly augmented in keratoconus, but also does the anterior chamber sagittal depth to anterior surface of the lens. The latter being only explained with limbal-scleral changes unrelated to cornea curvature [39].

Even in severe panuveitic syndromes, such as Vogt-Koyanagi-Harada (VKH), does ACD have its usefulness. ACD is decreased in the acute uveitic stage of VKH, consequent of the grand ciliary body oedema. Otsuki et al. measured the ACD in VHK after steroid pulse therapy. Effectively demonstrating that the anterior chamber is the fastest changing parameter, beginning at week 1 pulse therapy. Whereas $\mathrm{AL}$, spherical equivalent, BCVA and flare took at least 4 weeks to have statistically significant change. In addition, they concluded that ACD was the most sensitive parameter in determining anterior chamber inflammation, even more so that the standard cell and flare grading method [40].

\section{IOL Formula Considerations}

Gökce et al. assessed the accuracy of several IOL calculation formulas in normal axial length and discovered significant postoperative variations depending on the formula and the ACD. It was found that in ACD $\leq$ $3 \mathrm{~mm}$, the Barrett Universal formula was most precise and gave the least refractive error [41]. Although Hoffer Q may also be appropriate in this setting [42]. In the 3-3.5 $\mathrm{mm}$ ACD range, all the formulas were precise, the small differences between each other hand no statistical significance, thus Holladay 1, Holladay 2, Hoffer $Q$, SRK/T, Haigis, Hill-RBF, Barret Universal 11 and OLCR may be appropriate for this $A C D$ in normal AL patients. For $A C D \geq 3.5 \mathrm{~mm}$ three formulas had the lowest mean refractive error. They were Barret Universal 11, Haigis, and Holladay 2. Although, Barret Universal $\mathbf{1 1}$ and Haigis had better performance than the best $[41,42]$. For patients with $A L \leq 22 \mathrm{~mm}$ plus $A C D<2.4 \mathrm{~mm}$, the most precise IOL formula to date is Barret Universal II, Haigis [42], nevertheless, the latter had a wider error range.

\section{Disclosure}

The authors report no conflict of interest in this work.

\section{References}

1. Hashemi H, Yekta A, Khodamoradi F, Aghamirsalim M, Asharlous A, et al. (2019) Anterior chamber indices in a population based study using the Pentacam. Int Ophthalmol 39: 2033-2040.

2. Shrivastava AK, Behera P, Kacher R, Kumar B (2019) Effect of anterior chamber depth on predictive accuracy of seven intraocular lens formulas in eyes with axial length less than $22 \mathrm{~mm}$. Clin Ophthalmol 13: 1579-1586.

3. Maggon R, Singh SK, Jha M, Mishra A, Gupta S, et al. (2019) Correlation between ocular axial length and anterior chamber depth and a differential analysis in same-sized eyes. Kerala J Ophthalmol 31: 28.

4. Ejimadu CS, Aprioku IN (2019) Analysis of Ocular Axial Length and Anterior Chamber Depth in Port Harcourt, Nigeria. World Journal of Ophthalmology \& Vision Research 2

5. Xu L, Cao WF, Wang YX, Chen CX, Jonas JB (2008) Anterior chamber depth and chamber angle and their associations with ocular and general parameters: the Beijing Eye Study. Am J Ophthalmol 145: 929-936.

6. Fernández-Vigo JI, Fernández-Vigo JÁ, Macarro-Merino A, Fernández-Pérez C, Martínez de-la-Casa JM, et al. (2016) Determinants of anterior chamber depth in a large Caucasian population and agreement between intra-ocular lens Master and Pentacam measurements of this variable. Acta Ophthalmol 94: e150-e155.

7. Rüfer F, Schröder A, Klettner A, Frimpong-Boateng A, Roider JB, et al. (2010) Anterior chamber depth and iridocorneal angle in healthy White subjects: effects of age, gender and refraction. Acta Ophthalmol 88: 885-890.

8. Sun JH, Sung KR, Yun S-C, Cheon MH, Tchah HW, et al. (2012) Factors associated with anterior chamber narrowing with age: an optical coherence tomography study. Invest Ophthalmol Vis Sci 53: 2607-2610.

9. Sng CC, Foo LL, Cheng CY, Allen JC, He M, et al. (2012) Determinants of anterior chamber depth: the Singapore Chinese Eye Study. Ophthalmology 119: 1143-1150.

10. Yan PS, Lin HT, Wang QL, Zhang ZP (2010) Anterior Segment Variations with Age and Accommodation Demonstrated by Slit-Lamp-Adapted Optical Coherence Tomography. Ophthalmology 117: 2301-2307.

11. Schuster AK, Pfeiffer N, Nickels S, Schulz A, Höhn R, et al. (2016) Distribution of Anterior Chamber Angle Width and Correlation With Age, Refraction, and Anterior Chamber 
Depth-The Gutenberg Health Study. Invest Ophthalmol Vis Sci 57: 3740-3746.

12. Shimizu Y, Nakakura S, Nagasawa T, Okamoto A, Tabuchi $\mathrm{H}$, et al. (2017) Comparison of the anterior chamber angle structure between children and adults. J AAPOS 21: 57- 62 .

13. Hoffer KJ, Savini G (2015) Anterior chamber depth studies. J Cataract Refract Surg 41: 1898-1904.

14. Shajari M, Cremonese $C$, Petermann K, Singh $P$, Müller M, et al. (2017) Comparison of Axial Length, Corneal Curvature, and Anterior Chamber Depth Measurements of 2 Recently Introduced Devices to a Known Biometer. Am J Ophthalmol 178: 58-64.

15. Sihota R, Kamble N, Sharma AK, Bhari A, Gupta A, et al. (2019) "Van Herick Plus": a modified grading scheme for the assessment of peripheral anterior chamber depth and angle. Br J Ophthalmol 103: 960-965

16. Nolan WP, See JL, Chew PTK, Friedman DS, Smit SD, et al. (2007) Detection of primary angle closure using anterior segment optical coherence tomography in Asian eyes. Ophthalmology 114: 33-39.

17. Mete A, Kimyon S, Saygılı O, Evişen A, Pamukcu C, et al. (2016) Dynamic changes in optic disc morphology, choroidal thickness, anterior chamber parameters, and intraocular pressure during Valsalva maneuver. Arq Bras Oftalmol 79: $209-213$

18. Taradaj K, Ginda T, Maciejewicz P, Ciechanowicz $P$, Suchonska B, et al. (2018) Pregnancy and the eye. Changes in morphology of the cornea and the anterior chamber of the eye in pregnant woman. Ginekol Pol. 89: 695-699.

19. Fieß A, Schuster AK, Nickels S, Urschitz MS, Elflein HM, et al. (2019) Association of Low Birth Weight With Altered Corneal Geometry and Axial Length in Adulthood in the German Gutenberg Health Study. JAMA Ophthalmol 137: 507-514.

20. Macias BR, Patel NB, Gibson CR, Samuels BC, Laurie SS, et al. (2020) Association of Long-Duration Spaceflight With Anterior and Posterior Ocular Structure Changes in Astronauts and Their Recovery. JAMA Ophthalmol 138: 553-559.

21. Ito S, Sairenchi T, Machida T, Takino $Y$, Kondo $Y$, et al (2019) Reduced aqueous humour ascorbic-acid concentration in women with smaller anterior chamber depth. Sci Rep 9: 372 .

22. Ma N, Siegfried C, Kubota M, Huang J, Liu Y, et al. (2016) Expression Profiling of Ascorbic Acid-Related Transporters in Human and Mouse Eyes. Invest Ophthalmol Vis Sci 57: 3440-3450.

23. Wang D, Qi M, He M, Wu L, Lin S (2012) Ethnic difference of the anterior chamber area and volume and its association with angle width. Invest Ophthalmol Vis Sci 53: 3139-3144.

24. Klein AP, Suktitipat B, Duggal P, Lee KE, Klein R, et al. (2009) Heritability analysis of spherical equivalent, axial length, corneal curvature, and anterior chamber depth in the Beaver Dam Eye Study. Arch Ophthalmol 127: 649-655.

25. Saw SM, Wong TY, Ting S, Foong AWP, Foster PJ (2007) The relationship between anterior chamber depth and the presence of diabetes in the Tanjong Pagar Survey. Am J Ophthalmol 144: 325-326.

26. Chansangpetch S, Tran B, Perez Cl, Siguan-Bell C, Lau K, et al. (2018) Comparison of Anterior Segment Optical Coherence Tomography Parameters Among Vietnamese, Chinese, and Whites. Am J Ophthalmol 195: 72-82.

27. Lozano J, de Alba MA, Lozano JF, Mendoza G, Tamez A, et al. (2014) Anterior Chamber Depth in Hispanic Population. Invest Ophthalmol Vis Sci 55: 1579-1579.
28. Muzyka-Woźniak M, Ogar A (2016) Anterior chamber depth and iris and lens position before and after phacoemulsification in eyes with a short or long axial length. $\mathrm{J}$ Cataract Refract Surg 42: 563-568.

29. Ning X, Yang Y, Yan H, Zhang J (2019) Anterior chamber depth - a predictor of refractive outcomes after age-related cataract surgery. BMC Ophthalmol 19: 134.

30. Khalid M, Ameen SS, Ayub N, Mehboob MA (2019) Effects of anterior chamber depth and axial length on corneal endothelial cell density after phacoemulsification. Pak J Med Sci Q 35: 200-204.

31. Reuschel A, Bogatsch H, Oertel N, Wiedemann R (2015) Influence of anterior chamber depth, anterior chamber volume, axial length, and lens density on postoperative endothelial cell loss. Graefes Arch Clin Exp Ophthalmol 253: 745-752.

32. Alqudah AA, Bauer AJ, Straiko M, Terry MA (2019) Endothelial keratoplasty: The relationship between recipient anterior chamber depth and endothelial cell loss. Medicine 98: e16171.

33. Gür Güngör S, Akman A, Asena L, Aksoy M, Sarıgül Sezenöz A (2016) Changes in Anterior Chamber Depth after Phacoemulsification in Pseudoexfoliative Eyes and their Effect on Accuracy of Intraocular Lens Power Calculation. Turk J Ophthalmol 46: 255-258.

34. Fallah Tafti MR, Abdollah Beiki H, Mohammadi SF, Latifi G, Ashrafi E, et al. (2017) Anterior Chamber Depth Change Following Cataract Surgery in Pseudoexfoliation Syndrome; a Preliminary Study. J Ophthalmic Vis Res 12: 165-169.

35. Adewara BA, Adegbehingbe BO, Onakpoya $\mathrm{OH}$, Ihemedu CG (2018) Relationship between intraocular pressure, anterior chamber depth and lens thickness in primary open-angle glaucoma patients. Int Ophthalmol 38: 541-547.

36. Lee H, Zukaite I, Juniat V, Dimitry ME, Lewis A, et al. (2019) Changes in symmetry of anterior chamber following routine cataract surgery in non-glaucomatous eyes. Eye Vis (Lond) 6: 19.

37. Alvani A, Pakravan M, Esfandiari H, Safi S, Yaseri M, et al. (2016) Ocular Biometric Changes after Trabeculectomy. J Ophthalmic Vis Res 11: 296-303.

38. Wu SC, Lee YS, Wu WC, Chang SHL (2016) Anterior chamber depth and angle-closure glaucoma after central retinal vein occlusion. BMC Ophthalmol 16: 68 .

39. Mas-Aixala E, Gispets J, Lupón N, Cardona G (2018) Anterior chamber parameters in early and advanced keratoconus. A meridian by meridian analysis. Cont Lens Anterior Eye 41: 538-541.

40. Otsuki T, Shimizu K, Igarashi A, Kamiya K (2010) Usefulness of anterior chamber depth measurement for efficacy assessment of steroid pulse therapy in patients with VogtKoyanagi-Harada disease. Jpn J Ophthalmol 54: 396-400.

41. Gökce SE, Montes De Oca I, Cooke DL, Wang L, Koch DD, et al. (2018) Accuracy of 8 intraocular lens calculation formulas in relation to anterior chamber depth in patients with normal axial lengths. J Cataract Refract Surg 44: 362-368.

42. Yang S, Whang WJ, Joo CK (2017) Effect of anterior chamber depth on the choice of intraocular lens calculation formula. PLoS One 12: e0189868.
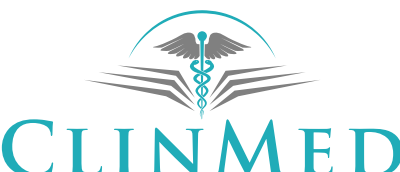

INTERNATIONAL LIBRARY 